\title{
THE DISTRIBUTION OF THE RH(D) BLOOD TYPES IN JAPAN ${ }^{1}$
}

\author{
Yoshiko Fujita, Katumi Tanaka, and Masako Tanimura \\ Department of Human Genetics, Tokyo Medical \\ and Dental University, Tokyo
}

\begin{abstract}
Summary Data on the Rh(D) types of 2,745,618 individuals were collected from prefectural health departments, health centers, and Red Cross blood centers in 35 of 47 prefectures in the whole country. Phenotypic and gene frequencies for each subpopulations were calculated and tabulated.

The frequency of $\mathrm{Rh}(\mathrm{D})$ negatives in the whole sample is $0.5428 \%$ and the frequency of the $\mathrm{Rh}(\mathrm{D})$ negative gene is estimated to be $7.37 \pm 0.03 \%$. An insignificant cline of the frequencies is suggested, i.e. the $R h(D)$ negative gene is increased from the northeast to the southwest of Japan.
\end{abstract}

\section{INTRODUCTION}

Frequencies of $\mathrm{Rh}$ blood types in different localities in Japan were summarized by Akaishi and Kudo (1975) who compiled available data on 10 prefectures from literature and through their own surveys. A majority of the studied prefectures cluster in the northmost part of the country and, therefore, distribution of the Rh system in the whole of Japan was still uncertain.

Recently, examination of the $\mathrm{Rh}(\mathrm{D})$ type is extensively carrying out at health centers and blood centers in the whole country. The present authors collected such data and report here the results of the analyses.

\section{MATERIALS AND METHODS}

The data on the numbers of Rh(D) positives and negatives were obtained from health centers, prefectural health departments and Red Cross blood centers by mail. Most data from Kagoshima Prefecture became available through Prof. Tetuo Zyo, Kagoshima University. Thus, data on the distribution of $\mathrm{Rh}(\mathrm{D})$ negatives were available from 35 of 47 prefectures for $2,745,618$ individuals, in total.

The blood-typing was carried out during the period from 1964 to 1975, mostly from 1970 to 1973.

Received March 2, 1978.

${ }^{1}$ This study was supported by the Scientific Research Grant from the Ministry of Education, Science and Culture of Japan No. 037020 in 1975, and No. 137071 in 1976 and 1977. 
Frequency of the $R h(D)$ negative allele was calculated simply by $d=\sqrt{n_{d} / N}$, where $n_{\downarrow}$ denotes the number of $R h(D)$ negative individuals and $N$ the size of the sample.

\section{RESULTS}

The phenotypic frequency of $\mathrm{Rh}(\mathrm{D})$ negative individuals in the entire sample was $0.5428 \%$ and frequency of the $R h(D)$ negative allele was estimated to be $7.37 \pm$

Table 1. Distribution of Rh(D) negatives in Japan for prefectures.

\begin{tabular}{|c|c|c|c|c|c|}
\hline \multirow{2}{*}{ Prefecture } & \multirow{3}{*}{$\begin{array}{c}\begin{array}{c}\text { Number } \\
\text { examined } \\
N\end{array} \\
31,203\end{array}$} & \multicolumn{2}{|c|}{ Rh(D) negative } & \multicolumn{2}{|c|}{ Gene frequency $(\%)$} \\
\hline & & \multirow{2}{*}{$\frac{\mathrm{n}_{\mathrm{d}}}{91}$} & \multirow{2}{*}{$\frac{\%}{0.2916}$} & \multirow{2}{*}{$\begin{array}{c}\mathrm{d} \\
5.40\end{array}$} & \multirow{2}{*}{$\frac{\begin{array}{c} \pm \text { Standard } \\
\text { error }\end{array}}{0.28}$} \\
\hline 2. Aomori & & & & & \\
\hline 4. Miyagi & 87,080 & 215 & 0.2469 & 4.97 & 0.17 \\
\hline 6. Yamagata & 41,516 & 226 & 0.5444 & 7.38 & 0.24 \\
\hline 8. Ibaraki & 45,916 & 385 & 0.8385 & 9.16 & 0.23 \\
\hline 9. Tochigi & 91 & 0 & & & \\
\hline 10. Gunma & 112 & 3 & & & \\
\hline 11. Saitama & 448 & 3 & & & \\
\hline 12. Chiba & 6,820 & 30 & 0.4399 & 6.63 & 0.60 \\
\hline 13. Tokyo & 293,688 & 1,491 & 0.5077 & 7.13 & 0.09 \\
\hline 14. Kanagawa & 7,832 & 45 & 0.5746 & 7.58 & 0.56 \\
\hline 15. Niigata & 39,155 & 210 & 0.5363 & 7.32 & 0.25 \\
\hline 16. Toyama & 10 & 0 & & & \\
\hline 18. Fukui & 187,714 & 1,006 & 0.5359 & 7.32 & 0.11 \\
\hline 19. Yamanashi & 11,025 & 76 & 0.6893 & 8.30 & 0.47 \\
\hline 20. Nagano & 348,946 & 1,952 & 0.5594 & 7.48 & 0.08 \\
\hline 21. Gifu & 4,578 & 25 & 0.5461 & 7.39 & 0.74 \\
\hline 22. Shizuoka & 1,501 & 12 & 0.7995 & 8.94 & 1.29 \\
\hline 23. Aichi & 90,582 & 405 & 0.4471 & 6.69 & 0.17 \\
\hline 24. Mie & 119,461 & 736 & 0.6161 & 7.85 & 0.14 \\
\hline 26. Kyoto & 11,610 & 52 & 0.4479 & 6.69 & 0.46 \\
\hline 27. Osaka & 604,866 & 3,385 & 0.5596 & 7.48 & 0.06 \\
\hline 28. Hyogo & 43,632 & 234 & 0.5363 & 7.32 & 0.24 \\
\hline 30. Wakayama & 6,492 & 57 & 0.8780 & 9.37 & 0.62 \\
\hline 31. Tottori & 99,371 & 474 & 0.4770 & 6.91 & 0.16 \\
\hline 32. Shimane & 106 & 0 & & & \\
\hline 34. Hiroshima & 3,669 & 42 & 1.1447 & 10.70 & 0.82 \\
\hline 35. Yamaguchi & 828 & 11 & & & \\
\hline 37. Kagawa & 23,611 & 100 & 0.4235 & 6.51 & 0.32 \\
\hline 38. Ehime & 8,094 & 44 & 0.5436 & 7.37 & 0.55 \\
\hline 39. Kochi & 69,559 & 487 & 0.7001 & 8.37 & 0.19 \\
\hline 40. Fukuoka & 25,788 & 68 & 0.2637 & 5.14 & 0.31 \\
\hline 41. Saga & 50,244 & 258 & 0.5135 & 7.17 & 0.22 \\
\hline 42. Nagasaki & 46,732 & 239 & 0.5114 & 7.15 & 0.23 \\
\hline 44. Oita & 44,533 & 293 & 0.6579 & 8.11 & 0.24 \\
\hline 46. Kagoshima & 388,805 & 2,249 & 0.5784 & 7.61 & 0.08 \\
\hline Total & $2,745,618$ & 14,904 & 0.5428 & 7.37 & 0.03 \\
\hline
\end{tabular}




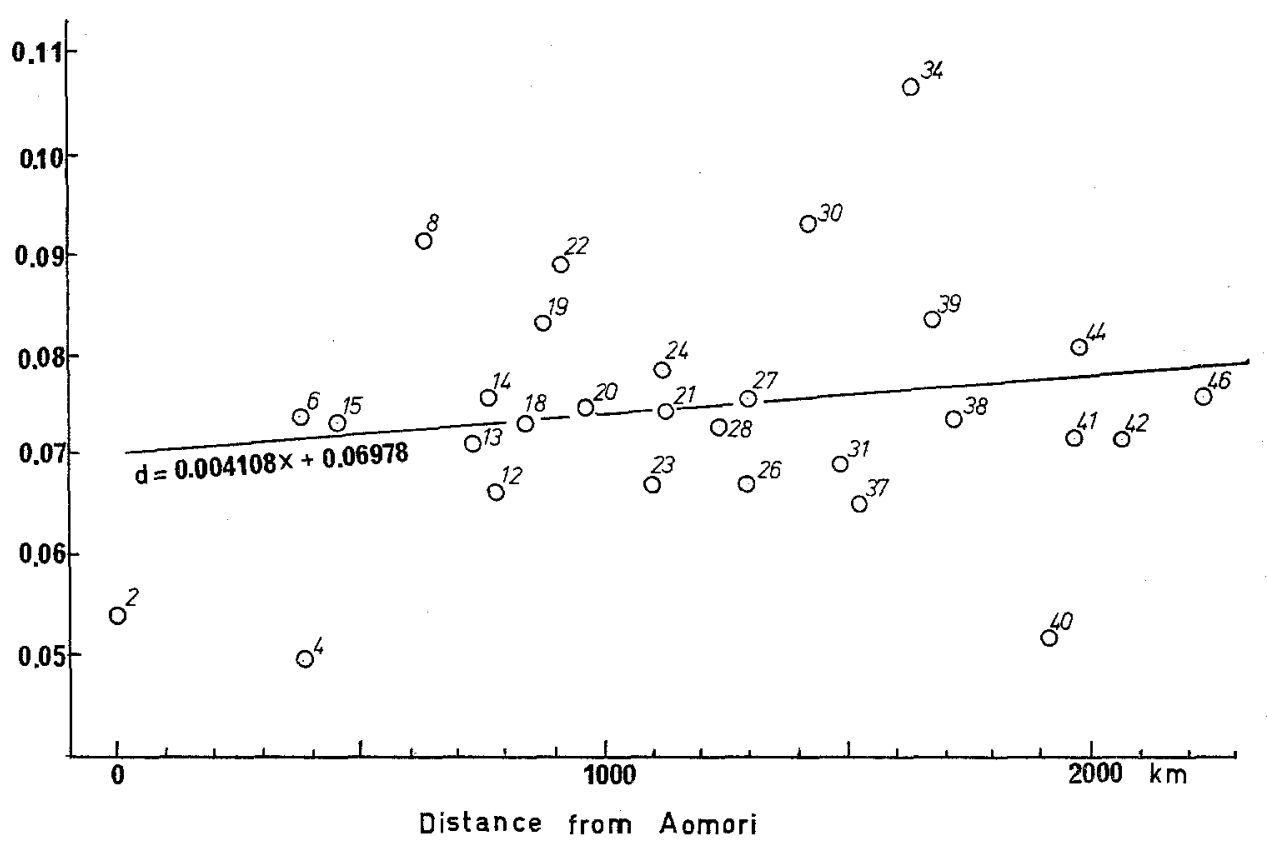

Fig. 1. Gradient of the frequency of the $R h(D)$ negative allele, d.

$0.03 \%$. The frequencies for prefectures are shown in Table 1 and those for health centers and districts in each prefecture are listed in the appendix. Each number of prefecture in italic and health center or district in roman corresponds to that shown in maps (Figs. 1 and 6-14) in a previous paper on the distribution of the ABO system (Fujita et al., 1978).

The frequency of the $\mathrm{Rh}(\mathrm{D})$ negative gene for each prefecture and the distance measured from Aomori, the northernmost of the Honshu Island, to each prefecture capital along the National Railroad were plotted in Fig. 1, which suggests a slight increase of the gene frequency with the increasing distance. An analysis of the data on 29 prefectures, excluding 6 prefectures with sample sizes smaller than 1,000 , revealed that the correlation coefficient between the distance from Aomori and the frequency of $\mathrm{Rh}(\mathrm{D})$ negative gene is 0.193 , which deviates from zero insignificantly $(t=1.022$ with 27 degrees of freedom, $0.3<P<0.4)$. The regression equation of the gene frequency on the distance in 1,000 kilometers, $x$, is $d=0.004108 x+0.06978$.

\section{DISCUSSION}

The frequency of the $\mathrm{Rh}(\mathrm{D})$ negatives in the whole sample is not greatly different from those reported by previous authors in various populations in Japan, excluding the Rhukyuan and the Ainu (see Akaishi and Kudo, 1975). As shown in Table 1, the frequency of $\mathrm{Rh}(\mathrm{D})$ negative gene for prefecture ranges from zero to $10 \%$ or higher. 
Such a wide variation is mostly attributable to the smallness of the sample sizes. The range in 6 prefectures with samples larger than 100,000 is as narrow as $7.13-$ $7.85 \%$.

On the other hand, it was suggested that the frequency of the $\mathrm{Rh}(\mathrm{D})$ negative gene was increased from the northeast to the southwest, though insignificantly. Such a cline may not be unexpected, since the frequencies of the $R h(D)$ types in the neighboring races are different from those in the Japanese and there are known significant clines of the frequencies of some polymorphic genes, including ABO blood group genes in Japan (Kobayasi, 1940; Tanaka, 1959; Nei and Imaizumi, 1966; Fujita et al., 1978). Frequency of the Rh(D) negative gene in Okinawa is apparently higher than that in other districts of Japan (Nakajima et al., 1967b; Waki, 1972). This may accord with the cline of the gene frequency which increases from the northeast to the southwest.

However, the Rh(D) negative gene is much more frequent in the Ainu (Kobayashi, 1953, 1956; Ishibashi, 1971; Misawa and Hayashida, 1972; Misawa et al., 1975), the original inhabitants in the northmost districts of Japan, and much rarer in Korean (Sarkisian, 1956; Won, 1960; Won et al., 1960; Mourant et al., 1976) and Taiwanese (Nakajima et al., 1967a, 1971) who have had close contacts particularly with the southwest districts of Japan. Thus, it may be premature to deduce a decisive explanation of the relationship between the distributions of $R h(D)$ negative gene in the Japanese and surrounding races.

Acknowledgment The authors are greatly indebted to health centers, prefectural health departments and Red Cross blood centers and their staffs, and Prof. T. Zyo, Kagoshima University Medical School, for the cordial cooperation of the collection of the data. They wish to express deepest appreciation to Dr. Hachiro Nakajima, Department of Legal Medicine, Tokyo Medical and Dental University, for valuable advices.

\section{REFERENCES}

Akaishi, S. and Kudo, T. 1975. Blood groups. In JIBP Synthesis: Human Adaptability, Vol. 2, Anthropological and Genetic Studies on the Japanese (S. Watanabe, S. Kondo, and E. Matsunaga, eds.), University of Tokyo Press, Tokyo, pp. 77-107.

Fujita, Y., Tanimura, M., and Tanaka, K. 1978. The distribution of the ABO blood groups in Japan. Jap. J. Human Genet. 23: 63-109.

Ishibashi, H. 1971. Distribution of ABO, MN, Q, Ss and Rh blood types among inhabitants in Hokkaido. Hokkaido J. Med. Sci. 46: 187-195 (in Japanese).

Kobayashi, H. 1953. On the blood groups of the Tokachi Ainu. Jap. J. Legal Med. 7: 223 (Abstract, in Japanese).

Kobayashi, H. 1956. On the blood groups of the Iburi Ainu. Jap. J. Legal Med. 10: 325-326 (Abstract, in Japanese).

Kobayasi, T. 1940. Statistische Beobachtung der Bluttypenverteilung in ABO-System bei Japanern (in Japanese). Hanzaigaku Zassi (Archiv für Gerichtliche Medizin und Kriminologie) 14: 727-735.

Misawa, S. and Hayashida, Y. 1972. On the blood groups of the Ainu in Hidaka, Hokkaido. The 56th Conf, of the Medico-Legal Soc, of Japan (Abstract, in Japanese). 
Misawa, S., Hayashida, Y., and Miki, T. 1975. Genetic composition of the Ainu: Blood groups. In JIBP Synthesis: Human Adaptability, Vol. 2, Anthropological and Genetic Studies on the Japanese (S. Watanabe, S. Kondo, and E. Matsunaga, eds.). University of Tokyo Press, Tokyo, pp. 265-273.

Mourant, A.E., Kopeć, A.C., and Domaniewska-Sobczak, K. 1976. The Distribution of Human Blood Groups and Other Polymorphisms, 2nd ed., p. 101 and pp. 351-505. Oxford Univ. Press.

Nakajima, H., Ohkura, K., Shen, Y-Z., Chow, Z-S., Lee, S-P., Orita, Y., Masuda, Y., and Takahara, S. 1967a. The distribution of several serological and biochemical traits in East Asia. I. The distribution of $\mathrm{ABO}, \mathrm{MN}, \mathrm{Q}$, Lewis and $\mathrm{Rh}$ groups in Taiwan. Jap. J. Human Genet. 11: 244-251.

Nakajima, H., Ohkura, K., Inafuku, S., Ogura, Y., Koyama, T., Hori, F., and Takahara, S. 1967b. The distribution of several serological and biochemical traits in East Asia. II. The distribution of ABO, MNSs, Q, Lewis, Rh, Kell, Duffy and Kidd blood groups in Rhukyu. Jap. J. Human Genet. 12: 29-37.

Nakajima, H., Ohkura, K., Huang, M-C., Saito, R., and Seto, T. 1971. The distribution of several serological and biochemical traits in East Asia. IV. The distribution of the blood groups in the Taiwanese mountain aborigines. Jap. J. Human Genet. 16: 57-68.

Nei, M. and Imaizumi, Y. 1966. Genetic structure of human populations. I. Local differentiation of blood group gene frequencies in Japan. Heredity 21: 9-35.

Sarkisian, S.S. 1956. Major blood groups in Koreans, U.S. Armed Forces Med. J. 7: 1320-1322.

Tanaka, T. 1959. A study on the Japanese from the standpoint of the blood groups (in Japanese with English summary). Hanzaigaku Zasshi (Jap. J. Legal Med. and Criminol.) 25: 37-67.

Waki, K. 1972. The distribution of the polymorphic groups of blood, serum protein and red cell enzyme among the inhabitants in Miyako and Yaeyama islands, Okinawa. Bull. Osaka Med. Sci. 6: 1-21 (in Japanese).

Won, C.D. 1960. $\mathrm{A}_{1} \mathrm{~A}_{2} \mathrm{BO}$ blood groups and Rh-Hr blood types in Koreans. 8th Congr. Int. Soc. Blood Transf., Tokyo: 170-173.

Won, C.D., Shin, H.S., Kim, S.W., Swandson, J., and Matson, A. 1960. Distribution of hereditary blood factors among Koreans residing in Seoul, Korea. Am. J. Phys. Anthropol. 18: 115-124. 
APPENDIX. DISTRIBUTION OF RH(D) NEGATIVES AND RH(D) NEGATIVE GENE IN JAPAN FOR HEALTH CENTERS OR DISTRICTS

\begin{tabular}{|c|c|c|c|c|c|}
\hline \multirow{2}{*}{$\begin{array}{l}\text { Health center } \\
\text { or district }\end{array}$} & \multirow{2}{*}{$\begin{array}{l}\text { Number } \\
\text { examined } \\
N\end{array}$} & \multicolumn{2}{|c|}{$\mathrm{Rh}(\mathrm{D})$ negative } & \multicolumn{2}{|c|}{ Gene frequency $(\%)$} \\
\hline & & $\mathrm{n}_{\mathrm{d}}$ & $\%$ & $\mathrm{~d}$ & \pm S.E. \\
\hline \multicolumn{6}{|l|}{ 2. Aomori } \\
\hline Total & 31,203 & 91 & 0.2916 & 5.40 & 0.28 \\
\hline \multicolumn{6}{|l|}{ 4. Miyagi } \\
\hline 5. Sendai-Higashi (似台東) & 1,703 & 3 & 0.1762 & 4.20 & 1.21 \\
\hline 6. Sendai-Minami (似台南) & 1,309 & 3 & 0.2292 & 4. 79 & 1.38 \\
\hline 7. Sendai-Kita（仙台北） & 1,262 & 0 & 0.0 & 0.0 & 1.41 \\
\hline Prefect. Health Dept. & 82,806 & 209 & 0.2524 & 5.02 & 0.17 \\
\hline Total & 87,080 & 215 & 0.2469 & 4.97 & 0.17 \\
\hline \multicolumn{6}{|l|}{ 6. Yamagata } \\
\hline 1. Yamagata (山形) & 8,029 & 49 & 0.6103 & 7.81 & 0.56 \\
\hline 2. Sagae（寒河江） & 5,919 & 25 & 0.4224 & 6.50 & 0.65 \\
\hline 3. Yonezawa (米沢) & 6,181 & 36 & 0.5824 & 7.63 & 0.63 \\
\hline Prefect. Health Dept. & 21,387 & 116 & 0.5424 & 7.36 & 0.34 \\
\hline Total & 41,516 & 226 & 0.5444 & 7.38 & 0.24 \\
\hline
\end{tabular}

8. Ibaraki

\begin{tabular}{lrrrrr} 
6. Omiya (大宮) & 448 & 4 & 0.8929 & 9.45 & 2.35 \\
Prefect. Health Dept. & 45,468 & 381 & 0.8380 & 9.15 & 0.23 \\
\hline Total & 45,916 & 385 & 0.8385 & 9.16 & 0.23
\end{tabular}

9. Tochigi

5. Ashikaga (足利) $91 \quad 0$

10. Gunma

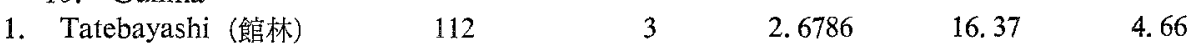

11. Saitama

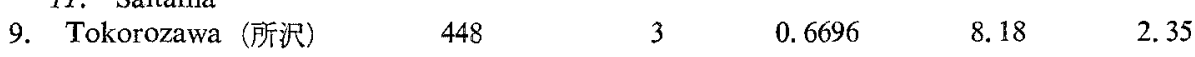

12. Chiba

\begin{tabular}{|c|c|c|c|c|c|}
\hline 1. Mobara (茂原) & 630 & 4 & 0.6349 & 7.97 & 1.99 \\
\hline 2. Sakura（佐倉） & 334 & 6 & 1.7964 & 13.40 & 2.71 \\
\hline 3. Chuo (中央) & 241 & 0 & 0.0 & 0.0 & 3.22 \\
\hline 4. Kamogawa (鴨川) & 1,155 & 4 & 0.3463 & 5.88 & 1.47 \\
\hline 5. Kashiwa (柏) & 1,706 & 5 & 0.2931 & 5.41 & 1.21 \\
\hline 7. Tateyama (館山) & 1,185 & 7 & 0.5907 & 7.69 & 1.45 \\
\hline 8. Noda (野田) & 1,569 & 4 & 0.2549 & 5.05 & 1.26 \\
\hline Total & 6,820 & 30 & 0.4399 & 6.63 & 0.60 \\
\hline
\end{tabular}

13. Tokyo

\begin{tabular}{|c|c|c|c|c|c|}
\hline Katsushika (暮飾) & 933 & 4 & 0.4287 & 6.55 & 1.63 \\
\hline 2. Edogawa (江户JI) & 996 & 4 & 0.4016 & 6.34 & 1.58 \\
\hline 3. Adachi (足立) & 2,546 & 4 & 0.1571 & 3.96 & 0.99 \\
\hline Sumida (墨田) & 1,404 & 8 & 0.5698 & 7.55 & 1.33 \\
\hline
\end{tabular}




\begin{tabular}{|c|c|c|c|c|c|c|}
\hline \multirow{2}{*}{\multicolumn{2}{|c|}{$\begin{array}{l}\text { Health center } \\
\text { or district }\end{array}$}} & \multirow{3}{*}{$\frac{\begin{array}{c}\text { Number } \\
\text { examined } \\
N\end{array}}{1,525}$} & \multicolumn{2}{|c|}{ Rh(D) negative } & \multicolumn{2}{|c|}{ Gene frequency $(\%)$} \\
\hline & & & \multirow{2}{*}{$\frac{\mathrm{n}_{\mathrm{d}}}{5}$} & \multirow{2}{*}{$\frac{\%}{0.3279}$} & \multirow{2}{*}{$\frac{d}{5.73}$} & \multirow{2}{*}{$\frac{ \pm \text { S.E. }}{1.28}$} \\
\hline 5. & Koto（江東） & & & & & \\
\hline 6. & Arakawa (荒川) & 3,954 & 5 & 0.1265 & 3.56 & 0.79 \\
\hline 7. & Taito (台東) & 2,162 & 8 & 0.3700 & 6.08 & 1.07 \\
\hline 8. & Chuo (中央) & 4,207 & 7 & 0.1664 & 4.08 & 0.77 \\
\hline 9. & Kita（北） & 1,730 & 12 & 0.6936 & 8.33 & 1.20 \\
\hline 10. & Bunkyo (文京) & 2,161 & 7 & 0.3239 & 5.69 & 1.07 \\
\hline 11. & Chiyoda（千代田） & 3,354 & 18 & 0.5367 & 7.33 & 0.86 \\
\hline 12. & Minato（港） & 4,068 & 11 & 0.2704 & 5.20 & 0.78 \\
\hline 13. & Itabashi（板穚） & 2,903 & 21 & 0.7234 & 8.51 & 0.92 \\
\hline 14. & Toshima (豊島) & 1,394 & 7 & 0.5022 & 7.09 & 1.34 \\
\hline 15. & Shinjuku (新宿) & 1,738 & 13 & 0.7480 & 8.65 & 1.19 \\
\hline 16. & Shibuya（橴谷） & 1,319 & 4 & 0.3033 & 5.51 & 1.37 \\
\hline 17. & Meguro（目黑） & 935 & 4 & 0.4278 & 6.54 & 1.63 \\
\hline 18. & Shinagawa（品川） & 1,990 & 9 & 0.4523 & 6.73 & 1.12 \\
\hline 19. & Ota (大田) & 5,554 & 22 & 0.3961 & 6.29 & 0.67 \\
\hline 20. & Nerima (練馬) & 1,382 & 3 & 0.2171 & 4.66 & 1.34 \\
\hline 21. & Nakano (中野) & 1,543 & 8 & 0.5185 & 7.20 & 1.27 \\
\hline 22. & Suginami (杉並) & 3,351 & 13 & 0.3879 & 6.23 & 0.86 \\
\hline 23. & Setagaya（世田谷） & 4,595 & 20 & 0.4353 & 6.60 & 0.74 \\
\hline 24. & Musashino（武蔵野） & 910 & $\mathbf{0}$ & 0.0 & 0.0 & 1.66 \\
\hline 25. & Mitaka (三鷹) & 1,010 & 3 & 0.2970 & 5.45 & 1.57 \\
\hline 26. & Musashi-Chofu（武蔵 & 淍布) 430 & 1 & 0.2326 & 4.82 & 2.41 \\
\hline 27. & Tanashi（田無） & 422 & 0 & 0.0 & 0.0 & 2.43 \\
\hline 28. & Koganei（小金井) & 1,266 & 3 & 0.2370 & 4.87 & 1.40 \\
\hline 29. & Kodaira (小平) & 1,475 & 20 & 1.3559 & 11.64 & 1.29 \\
\hline 30. & Fuchu（府中） & 388 & 4 & 1.0309 & 10.15 & 2.53 \\
\hline 31. & Machida (町田) & 1,314 & 6 & 0.4566 & 6.76 & 1. 38 \\
\hline 32. & Tachikawa（立川） & 416 & 4 & 0.9615 & 9.81 & 2.44 \\
\hline 33. & Hino（日野） & 396 & 2 & 0.5051 & 7.11 & 2. 51 \\
\hline 34. & Hachioji (八王子) & 1,084 & 8 & 0.7380 & 8.59 & 1.51 \\
\hline 35. & Ome（青梅） & 6,287 & 21 & 0.3340 & 5.78 & 0.63 \\
\hline 36. & Itsukaichi（五日市） & 1,045 & 3 & 0.2871 & 5.36 & 1.54 \\
\hline \multicolumn{2}{|c|}{ Subtotal } & 72,187 & 292 & 0.4045 & 6.36 & 0.19 \\
\hline \multicolumn{2}{|c|}{ Prefect. Health Dept. } & 101,436 & 539 & 0.5314 & 7.29 & 0.16 \\
\hline \multicolumn{2}{|c|}{ Others } & 120,065 & 660 & 0.5497 & 7.41 & 0.14 \\
\hline \multicolumn{2}{|c|}{ Grandtotal } & 293,688 & 1,491 & 0.5077 & 7.13 & 0.09 \\
\hline
\end{tabular}

\begin{tabular}{lrrrrr}
\multicolumn{1}{l}{ 14. Kanagawa } & & & & \\
1. Daishi (川崎大師) & 1,046 & 5 & 0.4780 & 6.91 & 1.54 \\
2. Saiwai (川崎幸) & 2,125 & 9 & 0.4235 & 6.51 & 1.08 \\
3. Nakahara (川猗中原) & 451 & 3 & 0.6652 & 8.16 & 2.35 \\
4. Takatsu (川崎高津) & 1,941 & 2 & 0.1030 & 3.21 & 1.13 \\
6. Midori (横浜緑) & 1,430 & 19 & 1.3287 & 11.53 & 1.31 \\
9. Kanazawa (横浜金沢) & 414 & 4 & 0.9662 & 9.83 & 2.45 \\
10. Yokosuka-Kita (横須賀北) 425 & 3 & 0.7059 & 8.40 & 2.42 \\
\hline \multicolumn{7}{l}{ Total } & 7,832 & 45 & 0.5746 & 7.58 & 0.56
\end{tabular}




\begin{tabular}{|c|c|c|c|c|c|c|}
\hline & \multirow{2}{*}{$\begin{array}{l}\text { Health center } \\
\text { or district }\end{array}$} & \multirow{2}{*}{$\begin{array}{c}\text { Number } \\
\text { examined } \\
\mathrm{N}\end{array}$} & \multicolumn{2}{|c|}{ Rh(D) negative } & \multicolumn{2}{|c|}{ Gene frequency $(\%)$} \\
\hline & & & $\mathrm{n}_{\alpha}$ & $\%$ & $\mathrm{~d}$ & \pm S.E. \\
\hline & \multicolumn{6}{|l|}{ 15. Nigata } \\
\hline 2. & Niigata-Higashi（新潟束） & 15,990 & 65 & 0.4065 & 6.38 & 0.39 \\
\hline 3. & Niigata-Nishi（新潟西） & 7,440 & 51 & 0.6855 & 8.28 & 0.58 \\
\hline 4. & Maki (巻) & 804 & 0 & 0.0 & 0.0 & 1.76 \\
\hline 9. & Tokamachi（十日町） & 14,921 & 94 & 0.6300 & 7.94 & 0.41 \\
\hline \multicolumn{2}{|c|}{ Total } & 39,155 & 210 & 0.5363 & 7.32 & 0.25 \\
\hline
\end{tabular}

16. Toyama

2. Himi（水見）

$10 \quad 0$

18. Fukui

$\begin{array}{llllll}\text { Red Cross Blood Center } & 187,714 & 1,006 & 0.5359 & 7.32 & 0.11\end{array}$

19. Yamanashi

$\begin{array}{llllll}\text { Red Cross Blood Center } & 675 & 0 & 0.0 & 0.0 & 1.92\end{array}$

\begin{tabular}{llllll} 
Blood Center & 10,350 & 76 & 0.7343 & 8.57 & 0.49 \\
\hline Total & 11,025 & 76 & 0.6893 & 8.30 & 0.47
\end{tabular}

20. Nagano

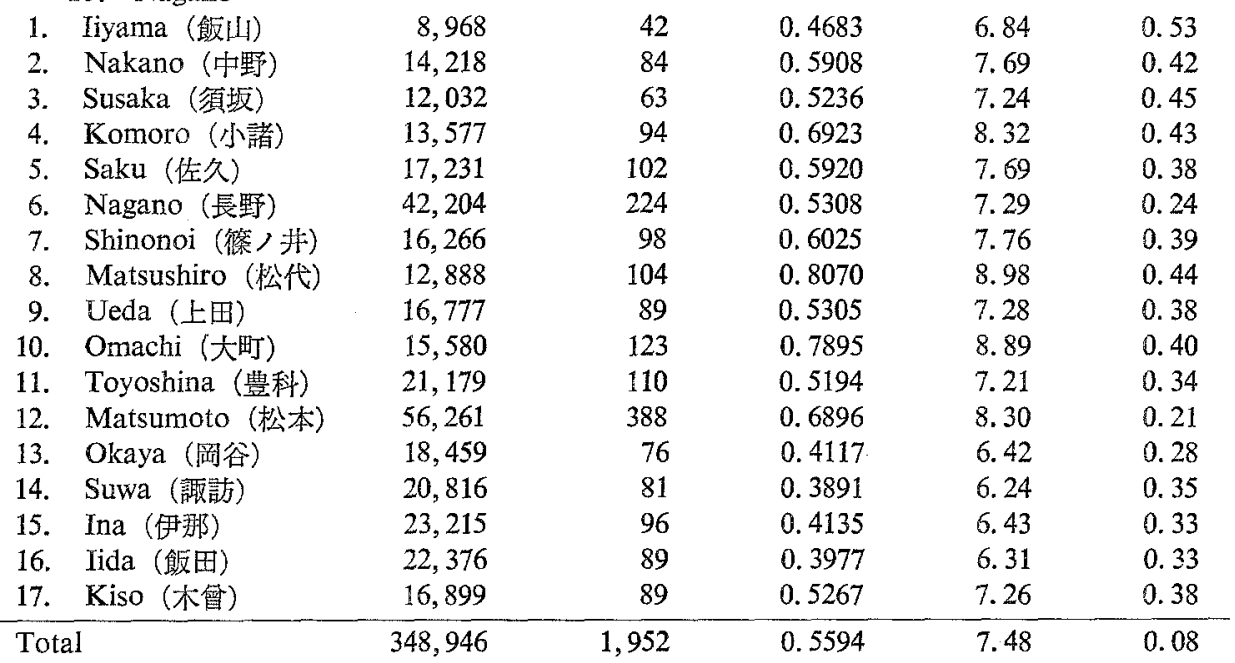

21. Gifu

\begin{tabular}{lrrrrr} 
1. Inaba (伊奈波) & 1,139 & 8 & 0.7024 & 8.38 & 1.48 \\
2. Ena (恵那) & 1,709 & 7 & 0.4096 & 6.40 & 1.21 \\
3. Gujo(郡上) & 391 & 4 & 1.0230 & 10.11 & 2.52 \\
4. Hajima (羽島) & 1,339 & 6 & 0.4481 & 6.69 & 1.36 \\
\hline Total & 4,578 & 25 & 0.5461 & 7.39 & 0.74
\end{tabular}




\begin{tabular}{|c|c|c|c|c|c|c|}
\hline & \multirow{2}{*}{$\begin{array}{l}\text { Health center } \\
\text { of district }\end{array}$} & \multirow{2}{*}{$\begin{array}{c}\text { Number } \\
\text { examined } \\
\mathrm{N}\end{array}$} & \multicolumn{2}{|c|}{ Rh(D) negative } & \multicolumn{2}{|c|}{ Gene frequency $(\%)$} \\
\hline & & & $\mathrm{n}_{\mathrm{d}}$ & $\%$ & $\mathrm{~d}$ & \pm S.E. \\
\hline & 22. Shizuoka & & & & & \\
\hline 2. & Shimoda (下田) & 679 & 2 & 0.2946 & 5.43 & 1.92 , \\
\hline 5. & Tenryu（天竜） & 69 & 0 & & & \\
\hline 6. & Hamamatsu (浜衿) & 753 & 10 & 1.3280 & 11.52 & 1.81 \\
\hline \multicolumn{2}{|c|}{ Total } & 1,501 & 12 & 0.7995 & 8.94 & 1.29 \\
\hline \multicolumn{7}{|c|}{ 23. Aichi } \\
\hline 1. & Shitara（設楽） & 2,835 & 16 & 0.5644 & 7.51 & 0.94 \\
\hline 2. & Shinshiro (新城) & 3,960 & 23 & 0.5808 & 7.62 & 0.79 \\
\hline 3. & Ichinomiya (一宮) & 4,694 & 21 & 0.4474 & 6.69 & 0.73 \\
\hline 4. & Toyokawa（豊川i) & 7,011 & 30 & 0.4279 & 6.54 & 0.60 \\
\hline 5. & Toyohashi (豊橋) & 3,214 & 18 & 0.5600 & 7.48 & 0.88 \\
\hline 6. & Tahara（田原） & 2,967 & 11 & 0.3707 & 6.09 & 0.92 \\
\hline 7. & Asuke（起助） & 1,852 & 6 & 0.3240 & 5.69 & 1.16 \\
\hline 8. & Gamagori（蒲郡） & 2,086 & 15 & 0.7191 & 8.48 & 1.09 \\
\hline 9. & Okazaki（岡崎） & 5,608 & 32 & 0.5706 & 7.55 & 0.67 \\
\hline 10. & Toyota（豊田) & 3,105 & 14 & 0.4509 & 6.71 & 0.90 \\
\hline 11. & Anjo (安城) & 2,572 & 14 & 0.5443 & 7.38 & 0.98 \\
\hline 12. & Nishio（西尾） & 1,294 & 1 & 0.0773 & 2. 78 & 1.39 \\
\hline 13. & Kariya (XIJ谷) & 11,783 & 35 & 0.2970 & 5.45 & 0.46 \\
\hline 14. & Handa (半田) & 6,613 & 29 & 0.4385 & 6.62 & 0.61 \\
\hline 15. & Mihama（美浜) & 277 & 0 & 0.0 & 0.0 & 3.00 \\
\hline 16 & Seto（瀬户） & 4,689 & 23 & 0.4905 & 7.00 & 0.73 \\
\hline 17. & Kasugai (春日井) & 4,484 & 17 & 0.3791 & 6.16 & 0.75 \\
\hline 18: & Nagoya (名古屋) & 697 & 3 & 0.4304 & 6.56 & 1.89 \\
\hline 19. & Chita (知多) & 3,964 & 17 & 0.4289 & 6.55 & 0.79 \\
\hline 20. & Konan（江南） & 3,173 & 15 & 0.4727 & 6.88 & 0.89 \\
\hline 21. & Nishibiwajima (西枇杷島) & 5) 5,194 & 38 & 0.7316 & 8.55 & 0.69 \\
\hline 22. & Inazawa (稲沢) & 2,045 & 2 & 0.0978 & 3. 13 & 1. 11 \\
\hline 23. & Bisai (尾西) & 2,107 & 18 & 0.8543 & 9.24 & 1.08 \\
\hline 24. & Tsushima (津島) & 4,358 & 7 & 0.1606 & 4. 01 & 0.76 \\
\hline \multicolumn{2}{|c|}{ Total } & 90,582 & 405 & 0.4471 & 6.69 & 0.17 \\
\hline \multicolumn{7}{|c|}{. } \\
\hline \multicolumn{7}{|c|}{ 24. Mie } \\
\hline \multicolumn{2}{|c|}{ Preventive Med. Center } & 119,461 & 736 & 0.6161 & 7.85 & 0.14 \\
\hline \multicolumn{7}{|c|}{ 26. Kyoto } \\
\hline 1. & Maizuru（舞鶴） & 5,036 & 35 & 0.6947 & 8.33 & 0.70 \\
\hline 2. & Amino（㸚野） & 35 & 0 & & & \\
\hline 3. & Yamashina（山科） & 1,671 & 5 & 0.2992 & 5.47 & 1.22 \\
\hline 4. & Higashiyama（東山） & 2,012 & 6 & 0.2982 & 5.46 & 1.11 \\
\hline 5. & Fushimi（优見） & 410 & 2 & 0.4878 & 6.98 & 2.46 \\
\hline 6. & Nakakyo（中京） & 1,410 & 2 & 0.1418 & 3.77 & 1. 33 \\
\hline 7. & Shimokyo (下京) & 1,036 & 2 & 0.1931 & 4.39 & 1.55 \\
\hline \multicolumn{2}{|c|}{ Total } & 11,610 & 52 & 0.4479 & 6.69 & 0.46 \\
\hline
\end{tabular}




\begin{tabular}{|c|c|c|c|c|c|c|}
\hline \multirow{2}{*}{\multicolumn{2}{|c|}{$\begin{array}{l}\text { Health center } \\
\text { or district }\end{array}$}} & \multirow{2}{*}{$\begin{array}{l}\text { Number } \\
\text { examined } \\
\mathrm{N}\end{array}$} & \multicolumn{2}{|c|}{ Rh(D) negative } & \multicolumn{2}{|c|}{ Gene frequency $(\%)$} \\
\hline & & & $\mathrm{n}_{\mathbb{\alpha}}$ & $\%$ & d & $\pm S . E$. \\
\hline \multicolumn{7}{|c|}{ 27. Osaka } \\
\hline 1. & Hirakata（枚方） & 29,951 & 191 & 0.6377 & 7.99 & 0.29 \\
\hline 2. & Takatsuki（高梘） & 30,896 & 183 & 0.5923 & 7.70 & 0.28 \\
\hline 3. & lbaragi (茨木) & 29,715 & 146 & 0.4913 & 7.01 & 0.29 \\
\hline 4. & Neyagawa（侵屋川） & 17,940 & 98 & 0.5463 & 7.39 & 0.37 \\
\hline 5. & Shijonawate（四条㪞） & 35,918 & 218 & 0.6069 & 7.79 & 0.26 \\
\hline 6. & Moriguchi（守曰) & 37,771 & 247 & 0.6539 & 8.09 & 0.26 \\
\hline 7. & Kadoma（門真） & 1,977 & 7 & 0.3541 & 5.95 & 1. 12 \\
\hline 8. & Hiraoka (枚烊) & 33,936 & 178 & 0.5245 & 7.24 & 0.27 \\
\hline 9. & Fuse（布施） & 35,841 & 207 & 0.5776 & 7.60 & 0.26 \\
\hline 10. & Yao（八尾） & 36,283 & 197 & 0.5430 & 7.37 & 0.26 \\
\hline 11. & Fujiidera（藤井寺） & 42,699 & 222 & 0.5199 & 7.21 & 0.24 \\
\hline 12. & Tondabayashi（富田林) 2 & 23,563 & 138 & 0.5857 & 7.65 & 0.32 \\
\hline 13. & Sayama（狭山） & 8,749 & 44 & 0.5029 & 7.09 & 0.53 \\
\hline 14. & Suita（吹田） & 27,867 & 142 & 0.5096 & 7. 14 & 0.30 \\
\hline 15. & Ikeda（竾田） & 25,348 & 135 & 0.5326 & 7.30 & 0.31 \\
\hline 16. & Toyonaka（豊中） & 41,380 & 243 & 0.5872 & 7.66 & 0.24 \\
\hline 17. & Asahi-ku（大阪市旭区） & 6,340 & 37 & 0.5836 & 7.64 & 0.63 \\
\hline 18. & Joto-ku（城東区） & 2,771 & 8 & 0.2887 & 5.37 & 0.95 \\
\hline 19. & Higashinari-ku（苯成区） & 7,121 & 42 & 0.5898 & 7.68 & 0.59 \\
\hline 20. & Ikuno-ku（生野区） & 1,576 & 2 & 0.1269 & 3.56 & 1.26 \\
\hline 21. & Higashi-ku（菓区） & 563 & 10 & 1.7762 & 13.33 & 2.09 \\
\hline 22. & Minami-ku（南区） & 1,166 & 0 & 0.0 & 0.0 & 1.46 \\
\hline 23. & Bandai（万代診療所） & 15,656 & 74 & 0.4727 & 6.88 & 0.40 \\
\hline 24. & Kita-ku (北区) & 1,605 & 4 & 0.2492 & 4. 99 & 1. 25 \\
\hline 25. & Nishi-ku（西区） & 3,926 & 24 & 0.6113 & 7.82 & 0.80 \\
\hline 26. & Nishinari-ku（西成区） & 6,364 & 38 & 0.5971 & 7.73 & 0.62 \\
\hline 27. & Suminoe (住之江) & 774 & 2 & 0.2584 & 5.08 & 1.79 \\
\hline \multirow[t]{2}{*}{28.} & \multicolumn{6}{|l|}{ Nishiyodogawa-ku } \\
\hline & (西淀川沤) & 5,813 & 14 & 0.2408 & 4.91 & 0.65 \\
\hline 29. & Izumiotsu（㬌大津） & 21,325 & 144 & 0.6753 & 8.22 & 0.34 \\
\hline 30. & Izumi（覀泉） & 13,469 & 98 & 0.7276 & 8.53 & 0.43 \\
\hline 31. & Kishiwada（岸和田） & 20,588 & 118 & 0.5731 & 7.57 & 0.35 \\
\hline 32. & Kaizuka（貝塚) & 12,509 & 68 & 0.5436 & 7.37 & 0.45 \\
\hline 33. & Izumisano（泉佐野） & 10,696 & 51 & 0.4768 & 6.91 & 0.48 \\
\hline 34. & Ozaki (尾崎) & 12,770 & 55 & 0.4307 & 6.56 & 0.44 \\
\hline \multicolumn{2}{|c|}{ Total } & 604,866 & 3,385 & 0.5596 & 7.48 & 0.06 \\
\hline \multicolumn{7}{|c|}{ 28. Hyogo } \\
\hline 1. & Itami（伊丹） & 3,419 & 21 & 0.6142 & 7.84 & 0.85 \\
\hline 2. & Amagasaki-Kita（尼ヶ崎北 & 北) 399 & 5 & 1. 2531 & 11.19 & 2.49 \\
\hline 3. & Ibid & 344 & 2 & 0.5814 & 7.62 & 2.69 \\
\hline 4. & Amagasaki-Higashi & & 2 & 0.2946 & 5.43 & 1.92 \\
\hline 5. & 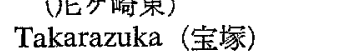 & 746 & 6 & 0.8043 & 8.97 & 1.82 \\
\hline 6. & Nishinomiya（西宮） & 4,178 & 21 & 0.5026 & 7.09 & 0.77 \\
\hline 7. & Ashiya（芦屋） & 440 & 6 & 1.3636 & 11.68 & 2.37 \\
\hline 8. & Santa（三田） & 732 & 2 & 0.2732 & 5.23 & 1.85 \\
\hline 9. & Nada（灘） & 94 & 0 & & & \\
\hline
\end{tabular}




\begin{tabular}{|c|c|c|c|c|c|c|}
\hline & \multirow{2}{*}{$\begin{array}{l}\text { Health center } \\
\text { or district }\end{array}$} & \multirow{2}{*}{$\begin{array}{l}\text { Number } \\
\text { examined } \\
\mathrm{N}\end{array}$} & \multicolumn{2}{|c|}{ Rh(D) negative } & \multicolumn{2}{|c|}{ Gene frequency $(\%)$} \\
\hline & & & $\mathrm{n}_{\mathfrak{d}}$ & $\%$ & $\mathrm{~d}$ & + S.E. \\
\hline 10. & Ikuta（生田） & 162 & 1 & 0.6173 & 7.86 & 3.92 \\
\hline 11. & Kobe-Kita（神戸北） & 63 & 0 & & & \\
\hline 12. & Hyogo (兵庫) & 78 & 0 & & & \\
\hline 13. & Nagata（長田） & 18 & 0 & & & \\
\hline 15. & Yashiro (社) & 4,192 & 20 & 0.4771 & 6.91 & 0.77 \\
\hline 16. & Akashi（明石） & 1,249 & 13 & 1.0408 & 10.20 & 1.41 \\
\hline 17. & Kakogawa（加古川） & 2,835 & 23 & 0.8113 & 9.01 & 0.94 \\
\hline 19. & Himeji-Nishi（姫路西） & 213 & 3 & 1.4085 & 11.87 & 3.40 \\
\hline 20. & Yamazaki（山崎） & 835 & 2 & 0.2395 & 4.89 & 1.73 \\
\hline 21. & Tatsuno (竜野) & 11,483 & 54 & 0.4703 & 6.86 & 0.47 \\
\hline 22. & Aioi（相生） & 720 & 6 & 0.8333 & 9.13 & 1.86 \\
\hline 23. & Sayo（佐用） & 1,822 & 6 & 0.3293 & 5.74 & 1.17 \\
\hline 24. & Ako（赤穂） & 3,679 & 18 & 0.4893 & 6.99 & 0.82 \\
\hline 26. & Kasumi（香住） & 949 & 11 & 1. 1591 & 10.77 & 1.61 \\
\hline 27. & Hamasaka (浜坂) & 2,291 & 5 & 0.2182 & 4.67 & 1.04 \\
\hline 28. & Tsuna (津名) & 1,104 & 4 & 0.3623 & 6.02 & 1. 50 \\
\hline 30. & Mihara (三原) & 908 & 3 & 0.3304 & 5.75 & 1.66 \\
\hline Tot & & 43,632 & 234 & 0.5363 & 7.32 & 0.24 \\
\hline
\end{tabular}

\section{Wakayama}

1. Chuo (中央)

31. Tottori

Total

32. Shimane

1. Kawamoto (UI本)

6,492

57

0.8780

9. 37

0.62

34. Hiroshima

4. Shobara (庄原)

5. Chiyoda (千代田)

99,371

474

0.4770

6.91

0. 16

6. Kaita (海田)

9. Hiroshima Health Lab. (広島衛生試験所)

$\begin{array}{lrrrrr}\text { 4. Shobara (庄原) } & 1,217 & 16 & 1.3147 & 11.47 & 1.42 \\ \text { 5. Chiyoda (千代田) } & 1,833 & 16 & 0.8729 & 9.34 & 1.16 \\ \begin{array}{l}\text { 6. Kaita (海田) } \\ \text { 9. Hiroshima Health Lab. }\end{array} & 613 & 1 & & & \\ & \text { (位島衛生試験所) }\end{array}$

35. Yamaguchi

1. Iwakuni (岩国)

4. Toyota (㤟田)

Total

(㤟田)

106

0

0.0

0.0

4. 86

\section{Kagawa}

\begin{tabular}{lrrrrr} 
3. Marugame (丸刍) & 992 & 2 & 0.2016 & 4.49 & 1.59 \\
Red Cross Blood Center & 22,619 & 98 & 0.4333 & 6.58 & 0.33 \\
\hline Total & 23,611 & 100 & 0.4235 & 6.51 & 0.32
\end{tabular}

38. Ehime
1. Mishima (三島)
708
1
0.1412
3.76
1.88 


\begin{tabular}{|c|c|c|c|c|c|c|}
\hline \multirow{2}{*}{\multicolumn{2}{|c|}{$\begin{array}{l}\text { Health center } \\
\text { or district }\end{array}$}} & \multirow{2}{*}{$\begin{array}{l}\text { Number } \\
\text { examined } \\
N\end{array}$} & \multicolumn{2}{|c|}{ Rh(D) negative } & \multicolumn{2}{|c|}{ Gene frequency $(\%)$} \\
\hline & & & $\mathrm{n}_{\mathfrak{d}}$ & $\%$ & d & \pm S.E. \\
\hline 2. & Niihama (新居浜) & 478 & 3 & 0.6276 & 7.92 & 2.28 \\
\hline 3. & Saijo (西条) & 102 & 2 & 1.9608 & 14.00 & 4.90 \\
\hline 4. & Nyugawa（壬生川） & 111 & 1 & 0.9009 & 9.49 & 4.72 \\
\hline 5. & Imabari (今治) & 675 & 5 & 0.7407 & 8.61 & 1.92 \\
\hline 6. & Kuma (久万) & 1,146 & 10 & 0.8726 & 9.34 & 1.47 \\
\hline 7. & Matsuyama (松山) & 750 & 2 & 0.2667 & 5.16 & 1.82 \\
\hline 9. & Nomura (野村) & 304 & 0 & 0.0 & 0.0 & 2.87 \\
\hline 10. & Osu (大洲) & 18 & 0 & & & \\
\hline 11. & Yawatahama (八幡浜) & 18 & 0 & & & \\
\hline 12. & Uwa (宇和) & 208 & 3 & 1.4423 & 12.01 & 3.44 \\
\hline 13. & Uwajima（宇和島） & 715 & 6 & 0.8392 & 9.16 & 1.86 \\
\hline & Misho (御荘) & 2,861 & 11 & 0.3845 & 6.20 & 0.93 \\
\hline \multicolumn{2}{|c|}{ Total } & 8,094 & 44 & 0.5436 & 7.37 & 0.55 \\
\hline \multirow{2}{*}{\multicolumn{2}{|c|}{$\begin{array}{l}\text { 39. Kochi } \\
\text { Red Cross Blood Center }\end{array}$}} & & & & & \\
\hline & & 69,559 & 487 & 0.7001 & 8.37 & 0.19 \\
\hline \multicolumn{7}{|c|}{ 40. Fukuoka } \\
\hline & Tobata (户畑) & 2,160 & 4 & 0.1852 & 4.30 & 1.07 \\
\hline 2. & Wakamatsu (若松) & 1,797 & 9 & 0.5008 & 7.08 & 1.18 \\
\hline 3. & Tagawa (田川) & 1,675 & 16 & 0.9552 & 9.77 & 1.22 \\
\hline 4. & Soeda (添田) & 557 & 2 & 0.3591 & 5.99 & 2.11 \\
\hline 5. & Ukiha (浮羽) & 1,559 & 9 & 0.5773 & 7.60 & 1.26 \\
\hline 6. & Onga (遠賀) & 8,696 & 11 & 0.1265 & 3.56 & 0.54 \\
\hline 7. & Munakata (宗像) & 7,329 & 13 & 0.1774 & 4.21 & 0.58 \\
\hline 8. & Kasuya (粕屋) & 521 & 4 & 0.7678 & 8.76 & 2.18 \\
\hline 10. & Yame (八女) & 1,494 & 0 & 0.0 & 0.0 & 1.29 \\
\hline \multicolumn{2}{|c|}{ Total } & 25,788 & 68 & 0.2637 & 5.14 & 0.31 \\
\hline \multicolumn{7}{|c|}{ 41. Saga } \\
\hline 1. & Tosu (鳥栖) & 3,734 & 21 & 0.5624 & 7.50 & 0.82 \\
\hline 2. & Kanzaki (神崎) & 3,469 & 12 & 0.3459 & 5.88 & 0.85 \\
\hline 3. & Saga (佐賀) & 14,225 & 75 & 0.5272 & 7.26 & 0.42 \\
\hline 4. & Ogi (小城) & 4,688 & 32 & 0.6826 & 8.26 & 0.73 \\
\hline 5. & Kashima (鹿島) & 4,357 & 15 & 0.3443 & 5.87 & 0.76 \\
\hline 6. & Takeo (武雄) & 7,822 & 36 & 0.4602 & 6.78 & 0.56 \\
\hline 7. & Karatsu（唐涟） & 6,834 & 32 & 0.4682 & 6.84 & 0.60 \\
\hline & Imari (伊万里) & 5,115 & 35 & 0.6843 & 8.27 & 0.70 \\
\hline \multicolumn{2}{|c|}{ Total } & 50,244 & 258 & 0.5135 & 7.17 & 0.22 \\
\hline \multicolumn{7}{|c|}{ 42. Nagasaki } \\
\hline & Obama (小浜) & 8,000 & 14 & 0.1750 & 4.18 & 0.56 \\
\hline 2. & Omura (大村) & 6,567 & 26 & 0.3959 & 6.29 & 0.62 \\
\hline 3. & Kita (北) & 25,071 & 161 & 0.6422 & 8.01 & 0.31 \\
\hline 4. & Yoshii (吉井) & 5,790 & 31 & 0.5354 & 7.32 & 0.66 \\
\hline & Arikawa (有川) & 1,304 & 7 & 0.5368 & 7.33 & 1.38 \\
\hline \multicolumn{2}{|c|}{ Total } & 46,732 & 239 & 0.5114 & 7.15 & 0.23 \\
\hline
\end{tabular}




\begin{tabular}{|c|c|c|c|c|c|c|}
\hline \multirow{2}{*}{\multicolumn{2}{|c|}{$\begin{array}{l}\text { Health center } \\
\text { or district }\end{array}$}} & \multirow{2}{*}{$\begin{array}{l}\text { Number } \\
\text { examined } \\
N\end{array}$} & \multicolumn{2}{|c|}{$\mathrm{Rh}(\mathrm{D})$ negative } & \multicolumn{2}{|c|}{ Gene frequency $(\%)$} \\
\hline & & & $\mathrm{n}_{d}$ & $\%$ & d & \pm S.E. \\
\hline \multicolumn{7}{|c|}{ 44. Oita } \\
\hline \multicolumn{2}{|c|}{ Prefect. Health Dept. } & 44,533 & 293 & 0.6579 & 8.11 & 0.24 \\
\hline \multicolumn{7}{|c|}{ 46. Kagoshima } \\
\hline 1. & Okuchi-city（大口市） & 9,211 & 31 & 0.3366 & 5.80 & 0.52 \\
\hline 2. & Kokubu-city (国分市) & 8,533 & 48 & 0.5625 & 7.50 & 0.54 \\
\hline 3. & Tarumi-city（垂水市） & 4,566 & 24 & 0.5256 & 7.25 & 0.74 \\
\hline 4. & Kanoya-city (鹿屋市) & 15,804 & 96 & 0.6074 & 7.79 & 0.40 \\
\hline \multicolumn{7}{|c|}{ 5. Kagoshima-city } \\
\hline & （鹿罗島市） & 141,933 & 828 & 0.5834 & 7.64 & 0.13 \\
\hline 6. & Ibusuki-city（指宿市） & 8,338 & 67 & 0.8036 & 8.96 & 0.55 \\
\hline 7. & Izumi-city（出水市） & 4,380 & 19 & 0.4338 & 6.59 & 0.75 \\
\hline 8. & Akune-city（阿久根市） & 9,766 & 41 & 0.4198 & 6.48 & 0.50 \\
\hline 9. & Sendai-city（川丙南） & 19,276 & 109 & 0.5655 & 7.52 & 0.36 \\
\hline \multicolumn{7}{|c|}{ 10. Kushikino-city } \\
\hline & (串木野市) & 11,031 & 75 & 0.6799 & 8.25 & 0.47 \\
\hline 11. & Kaseda-city (加世田市) & 4,305 & 27 & 0.6272 & 7.92 & 0.76 \\
\hline & Makurazaki-city & & & & & \\
\hline & （炕崎沛） & 14,275 & 74 & 0.5184 & 7.20 & 0.42 \\
\hline 13. & Soo-gun (售於郡) & 19,200 & 122 & 0.6354 & 7.97 & 0.36 \\
\hline 14. & Kimotsuki-gun (肝付郡) & 20,986 & 128 & 0.6100 & 7.81 & 0.34 \\
\hline 15. & Isa-gun (伊佐郡) & 2,572 & 10 & 0.3888 & 6.24 & 0.98 \\
\hline 16. & Aira-gun（姶良郡） & 27,433 & 158 & 0.5760 & 7.59 & 0.30 \\
\hline & Izumi-gun (出水郡) & 7,382 & 23 & 0.3116 & 5.58 & 0.58 \\
\hline 18. & Satsuma-gun（薩摩郡） & 11,169 & 58 & 0.5192 & 7.21 & 0.47 \\
\hline & Hioki-gun (日置郡) & 21,537 & 135 & 0.6269 & 7.92 & 0.34 \\
\hline & Kawanabe-gun (川辺郡) & 4,750 & 31 & 0.6526 & 8.08 & 0.72 \\
\hline 21. & Ibusuki-gun (揖宿郡) & 10,068 & 72 & 0.7151 & 8.46 & 0.50 \\
\hline 22. & Nishinoomote (西之表) & 6,370 & 39 & 0.6122 & 7.82 & 0.62 \\
\hline & Yakushima（星久島） & 3,919 & 23 & 0.5868 & 7.66 & 0.80 \\
\hline & Tokunoshima (徳之島) & 2,001 & 11 & 0.5497 & 7.41 & 1.11 \\
\hline \multicolumn{2}{|c|}{ Total } & 388,805 & 2,249 & 0.5784 & 7.61 & 0.08 \\
\hline
\end{tabular}

